\title{
Probing ETEX-II data set with inverse modelling
}

\author{
M. Krysta ${ }^{1,2, *}$, M. Bocquet ${ }^{1,2}$, and J. Brandt ${ }^{3}$ \\ ${ }^{1}$ Université Paris-Est, CEREA, Research and Teaching Centre in Atmospheric Environment, Joint Laboratory École \\ Nationale des Ponts et Chaussées/EDF R\&D, 6-8 avenue Blaise Pascal, 77455 Champs sur Marne, France \\ ${ }^{2}$ INRIA, Paris-Rocquencourt Research Centre, France \\ ${ }^{3}$ Department of Atmospheric Environment, National Environmental Research Institute, Aarhus University, Frederiksborgvej \\ 399, P.O. Box 358, 4000 Roskilde, Denmark \\ *currently at: Laboratoire Jean Kuntzmann, University of Grenoble, BP 53X, 38041 Grenoble cedex, France
}

Received: 20 December 2007 - Published in Atmos. Chem. Phys. Discuss.: 12 February 2008

Revised: 18 June 2008 - Accepted: 18 June 2008 - Published: 24 July 2008

\begin{abstract}
We give here an account on the results of source inversion of the ETEX-II experiment. Inversion has been performed with the maximum entropy method on the basis of non-zero measurements and in conjunction with a transport model POLAIR3D. The discrepancy scaling factor between the reconstructed and the true mass has been estimated to be equal to 7. The results contrast with the method's performance on the ETEX-I source. In the latter case its mass has been reconstructed with an accuracy exceeding $80 \%$. The large value of the discrepancy factor for ETEX-II could be ascribed to modelling difficulties, possibly linked not to the transport model itself but rather to the quality of the measurements.
\end{abstract}

\section{Introduction}

The European Tracer EXperiment (ETEX) was designed to evaluate forecast performance of dispersion models as well as readiness and communication capacity of European institutions in case of an accidental release of harmful substances to the atmosphere. The experiment consisted of two controlled releases of harmless and non-depositing inert tracers (perfluorocarbon compounds). Since the release term was controlled and thereby well-known, and since the deposition and chemistry processes were non-existing, the design of the experiment made it an excellent test on models of atmospheric transport and diffusion from point sources at continental scale.

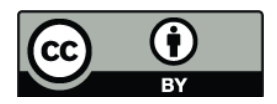

Correspondence to: M. Krysta

(monika.krysta@imag.fr)
The location of the two releases was in Monterfil, Brittany, France. The first release (ETEX-I) took place on 23 October 1994 and the second release (ETEX-II) some three weeks later, on 14 November 1994. Both experiments were carried out under comparable meteorological conditions with a low pressure system situated north of Scotland and westerly wind over the release site and over Western and Central Europe. In this paper we focus on the second ETEX release which took place between 15:00 UTC on 14 November and 02:45 UTC on 15 November 1994. $490 \mathrm{~kg}$ of PMCP (perfluoromethylcyclopentane) were released with a constant rate. In the first stage after the release a narrow cloud was advected rapidly eastwards (see the modelled plume in Fig. 1). Advection was driven by strong winds preceding a frontal passage which was linked with the low pressure area moving from the North Atlantic towards Scandinavia and Russia. The wind during ETEX-II was, however, much stronger compared to the ETEX-I episode, and the low pressure system over Western Europe was accompanied by rain. The experimental campaign coincided with a modelling exercise in which 28 models took part. Modelling turned out to be more difficult than in the case of the first release (Brandt, 1998).

The first ETEX experiment has been treated in many papers in which model results have been compared to observations, see (e.g., Brandt et al., 1998). The ultimate evaluation of the outcome of model intercomparison was presented in the final reports in 1998 (Mosca et al., 1998; Graziani et al., 1998a) where results from 49 models were included. In (Mosca et al., 1998) it was concluded that: "almost all the models show a satisfactory agreement with the measured values".

Published by Copernicus Publications on behalf of the European Geosciences Union. 


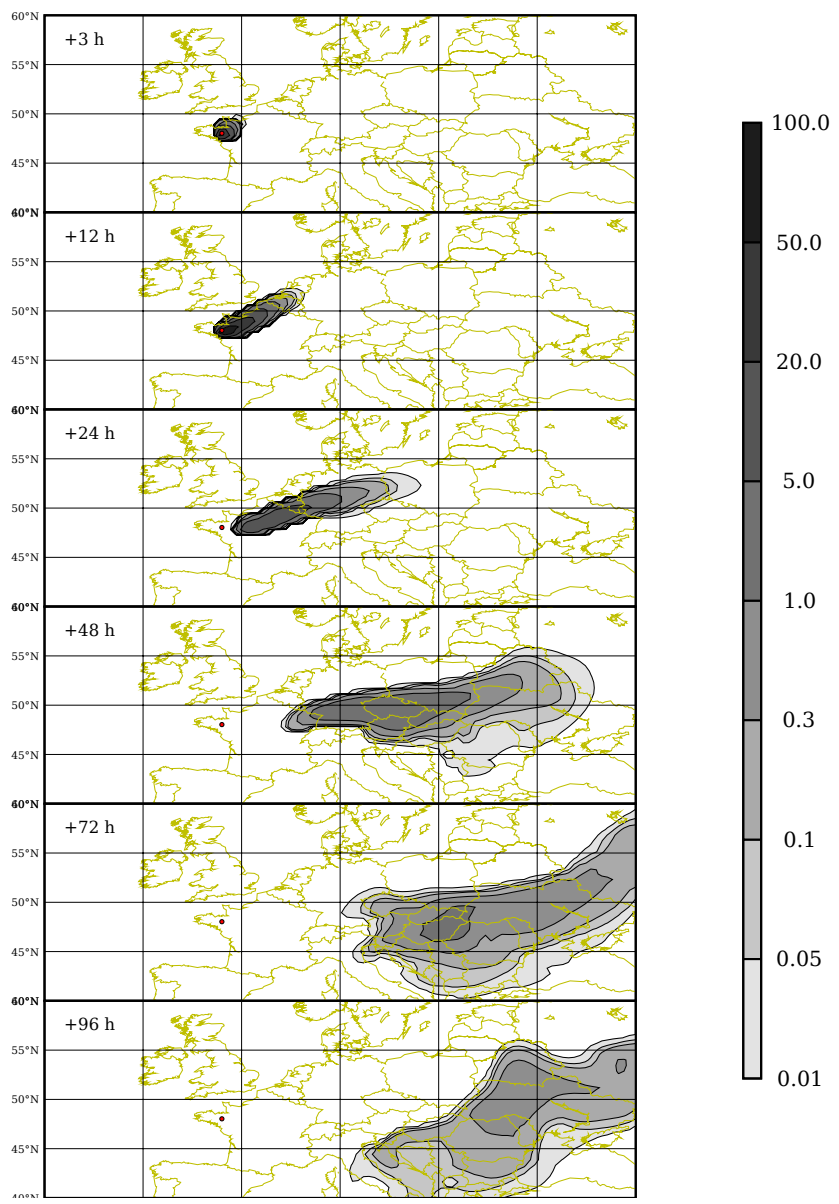

Fig. 1. Plume of the ETEX-II release simulated by POLAIR3D. The units are in $\mathrm{ng} \mathrm{m}^{-3}$. The snapshots' dates correspond to $3,12,24$, 28,72 , and $96 \mathrm{~h}$ after the start of the release.

Even though the meteorological conditions were quite similar, and the same measurement sites and procedures were used in both experiments, there was a significant difference in models' performance between ETEX-I and ETEX-II. In the latter case an overall bias of a model validated against the measurements is suggested to be of a factor of 10 (Brandt, 1998). The experiment's website (http://rem.jrc.ec.europa. eu/etex/) reports spatial and temporal analyses for a very limited number of time instants and monitoring stations. Figures of merit and time of arrival for some subsets of models are the only statistical indices disclosed by the JRC. In particular, a gap in the quality of model results for the two releases is underlined there. For the figure of merit in space (FMS) at $24 \mathrm{~h}$ after the release, half of the models exceeded $20 \%$ but only one of them reached the value of $40 \%$. In the case of the first ETEX release, an FMS above $40 \%$ was attained by 4 models out of 28 , and there were still models exceeding this value at $36 \mathrm{~h}$ and even $60 \mathrm{~h}$ after the release.

According to the detailed conclusions from the ETEX sym- posium in 1997 in Vienna, the following was stated about the ETEX-II release: "The second release showed larger discrepancies between observations and model results where all models significantly overpredicted surface concentrations after $12 \mathrm{~h}$ from the start of release. No clear explanation has yet been given for this result" (Nodop, 1997). The eventual evaluation of model intercomparison within ETEX-II was treated in the final report in 1998 (Graziani et al., 1998b) where results from the 28 models were presented. Some of the conclusions in this report stated: "Compared to the first release, much less tracer was found". It was also reported that: "Not only fewer non-zero values were observed, but also the concentration levels were much lower". Furthermore, according to the conclusions: "the cloud is apparently broken up into several parts moving at different speed in different directions. At many sites, the tracer was detected intermittently, rather than in a continuous sequence, as it might be expected". In the end, the report settles that: "The generally poor performance of the long-range dispersion models employed in the real-time simulation of the release are to put in relation with the complexity of the weather situation after the end of the second ETEX experiment".

Bad models' performance was considered rather strange among the modelling community because all the models performed satisfactorily with respect to ETEX-I. Since all the models showed a large overestimation with respect to ETEXII, the reason for this overprediction could be explained by one of the following two hypotheses: (1) there is a systematic error in all the model results e.g. due to the complexity of the weather situation which could result in a strong vertical mixing, or (2) there is a systematic error in the measurements which results in a significant negative bias (i.e. all the measurements are too small). The first hypothesis was concluded as a fact in (Graziani et al., 1998b). In this study, an inverse modelling procedure that was evaluated against ETEX-I with very good results (Bocquet, 2007), suggests that the second hypothesis is more likely to be true.

\section{Inverse modelling procedure}

We tackle in this work the reconstruction of the source of ETEX-II in order to check to what extent the retrieved temporal profile and the emitted mass agree with the true release. Source retrieval problem is addressed with the principle of maximum entropy on the mean. The method has been presented in (Bocquet, 2005a,c), and successfully applied to inversion of the source of ETEX-I (Bocquet, 2007) as well as the temporal and vertical profile of the source of the Chernobyl accident (Davoine and Bocquet, 2007). 


\subsection{Measurements}

There are 2248 valid measurements collected in the experiment. In contrast, however, with 969 non-zero values gathered in ETEX-I, only 476 measurements (12\% of all the measurements) resulting from the second release are non-null. Solely the latter subset has been used to perform inversion. The reason is our previous experience with the inversion method (for a non-Gaussian approach, assuming only positive sources) which made it clear that adding additional zero measurements is inefficient (Bocquet, 2007).

\subsection{Inversion method}

The retrieval of a source from measurements is an elaborate construct for the proper inversion of a source - measurement relationship. For a discrete formulation of the problem the relationship is of the form:

$\boldsymbol{\mu}=\boldsymbol{H} \boldsymbol{\sigma}+\boldsymbol{\varepsilon}$,

where $\sigma \in \mathbb{R}^{N}$ is a source vector, and $\boldsymbol{\mu} \in \mathbb{R}^{d}$ stands for the measurements. The matrix $\boldsymbol{H}$ (often described as the Jacobian matrix) embodies modelling of dispersion phenomenon as well as sampling of measurements. Therefore, $\boldsymbol{\varepsilon} \in \mathbb{R}^{d}$ represents a combined model-representativity-measurement error. Due to a large number of unknowns $N$ that we want to invert and a comparatively small number of measurements $d$, $\boldsymbol{H}$ has been computed in an adjoint mode.

\subsubsection{Duality test}

The modelling of the transport phenomenon for the needs of the inversion procedure is ensured by our Eulerian chemistry transport model POLAIR3D. It is equipped with a module devoted to radionuclides which has recently been validated on ETEX-I, Chernobyl and Algeciras releases (Quélo et al., 2007).

There are two ways of obtaining an adjoint associated with a discrete direct problem. One can discretise an adjoint of an original continuous model or adjoin an already discretised one. We have chosen here the first option which has the advantage to be simple. It boils down to time reversal of meteorological fields in a direct model. The latter possibility could have also been chosen since POLAIR3D has an extensively tested and employed adjoint module. In that case an agreement between the synthetic measurements obtained via the adjoint model, $c_{i}^{\text {adjoint }}$, Eq. (1) and the corresponding ones delivered by the direct simulation, $c_{i}^{\text {direct }}$ would have been precise. The precision being obviously up to computer's accuracy, provided the dispersion model is linear. Our choice of the adjoint implies a poorer match between the values of synthetic measurements computed in these two different ways. The discrepancy is shown in Fig. 2 and contrasts with a straight line that would have been obtained if we had used the adjoint of a discrete model. To estimate quantitatively

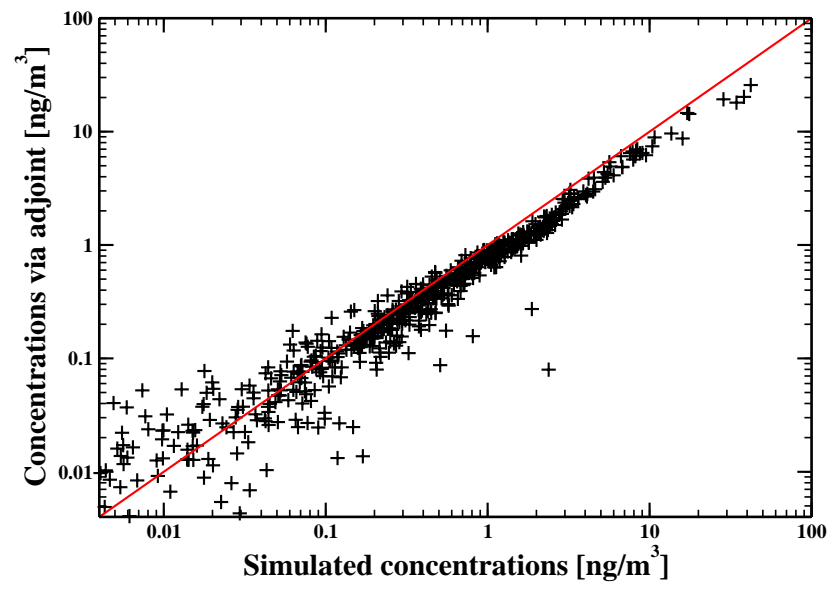

Fig. 2. Duality test. Measurements computed according to the measurement equation, Eq. (1) with $\boldsymbol{H}$ obtained in the dual mode (vertical axis) versus measurements obtained via sampling of a concentration field in a direct simulation (horizontal axis).

the discrepancies, we have computed the average fractional bias between both types of forecast concentration, $c_{i}^{\text {direct }}$ and $c_{i}^{\text {adjoint }}$ :

$\frac{2}{D} \sum_{i=1}^{D} \frac{\left|c_{i}^{\text {direct }}-c_{i}^{\text {adjoint }}\right|}{c_{i}^{\text {direct }}+c_{i}^{\text {adjoint }}}$.

Whenever both of them are below $0.01 \mathrm{ng} \mathrm{m}^{3}$, it has been assumed that they match perfectly (negligible concentration is assessed in both cases). The mismatch indicator computed with respect to all the measurements, $D=2248$, is greater then $20 \%$ for 366 measurements (that is to say for $16.3 \%$ of the concentrations), whereas 112 measurements (5\%) have a mismatch exceeding $50 \%$.

We deal in this paper, however, with an inversion of real measurements which are subject to measurement and modelling error, Eq. (1). In comparison to them, the error linked to the way the adjoint solutions have been computed can be regarded as negligible.

\subsubsection{Cost function}

The inversion method allows to take into account some prior knowledge which is appropriate to an accidental source. This capacity is of crucial importance due to scarcity of information on an accidental release and hence the necessity of making use of any available pieces of it. Prior information to be exploited refers in particular to positivity and boundedness of the source. Mathematical form of such information is encoded in a cost function arising from, for instance, Bernoulli law. However, any other positive prior law, such as an exponential law, would have served a decent purpose. The minimum of a cost function allows to shift from prior $(\nu(\sigma)$ for the source and $\zeta(\varepsilon)$ for the errors) to posterior probability 


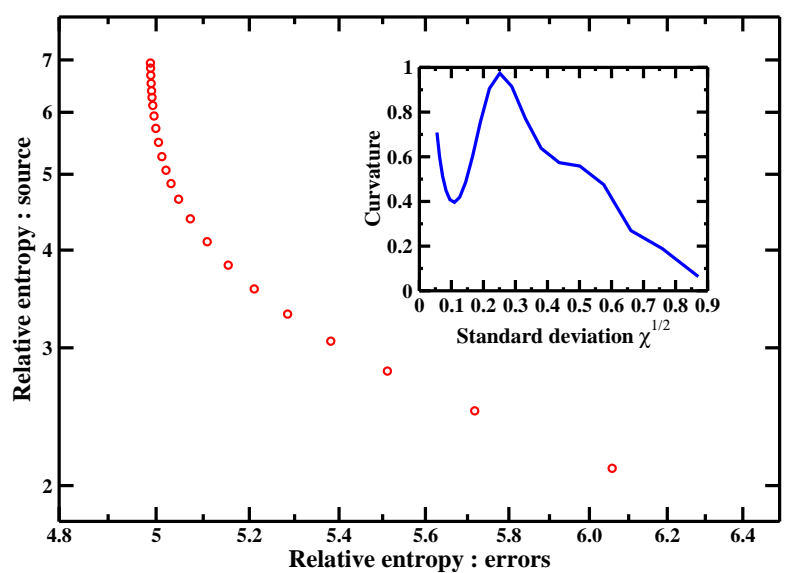

Fig. 3. L-curve for a varying standard deviation. Relative entropy for source and errors on the axes refer to $\mathcal{K}_{\boldsymbol{\sigma}}$ and $\mathcal{K}_{\boldsymbol{\varepsilon}}$ in Eq. (3). The point of inflection can only be identified thanks to the graph of curvature (inlay). Spatial resolution is $2.25^{\circ} \times 2.25^{\circ}$.

density function ( $p(\boldsymbol{\sigma})$ and $q(\boldsymbol{\varepsilon})$, respectively), and consequently defines the inverted source. Usually such a cost function is defined in the space of the discretised source $\sigma \in \mathbb{R}^{N}$ (and errors $\boldsymbol{\varepsilon} \in \mathbb{R}^{d}$ ):

$\mathcal{K}_{\boldsymbol{\sigma}, \boldsymbol{\varepsilon}}=\mathcal{K}_{\boldsymbol{\sigma}}+\mathcal{K}_{\boldsymbol{\varepsilon}}=\sum_{\boldsymbol{\sigma}} p(\boldsymbol{\sigma}) \ln \frac{p(\boldsymbol{\sigma})}{v(\boldsymbol{\sigma})}+\sum_{\boldsymbol{\varepsilon}} q(\boldsymbol{\varepsilon}) \ln \frac{q(\boldsymbol{\varepsilon})}{\zeta(\boldsymbol{\varepsilon})}$,

and minimised under the constraint, Eq. (1). Because the number of observations $(d=476)$ is much lower than the number of unknown variables $\left(N \simeq 20 \times 10^{3}\right)$, it is computationally efficient to trade a cost function in the state space of the sources to a cost function in a space isomorphic to the observation space. Mathematics authorises the trade on the basis of the convexity of the cost functions which is guaranteed by the maximum entropy on the mean approach. Ultimately, one deals with the following cost function (Bocquet, 2005b; Krysta and Bocquet, 2007):

$$
\begin{aligned}
\widehat{\mathcal{L}}(\boldsymbol{\beta}) & =\sum_{k=1}^{N} \ln \left\{1-\gamma_{k}+\gamma_{k} \exp \left(m_{k}\left[\boldsymbol{\beta}^{\dagger} \boldsymbol{H}\right]_{k}\right)\right\} \\
& +\frac{1}{2} \boldsymbol{\beta}^{\dagger} \boldsymbol{R} \boldsymbol{\beta}-\boldsymbol{\beta}^{\dagger} \boldsymbol{\mu},
\end{aligned}
$$

where $\boldsymbol{\beta} \in \mathbb{R}^{d}$ is the vector conjugated to the measurement vector $\boldsymbol{\mu} . \boldsymbol{R}$ is the observation error covariance matrix which parametrises the Gaussian prior law on the errors, $\zeta(\varepsilon)$. It is chosen diagonal, of the simplest form $\boldsymbol{R}=\chi \boldsymbol{I}$, with $\boldsymbol{I}$ the identity matrix in observation space. The estimated source and errors are then given, in terms of the minimum $\overline{\boldsymbol{\beta}}$ of $\widehat{\mathcal{L}}$, by the estimators:

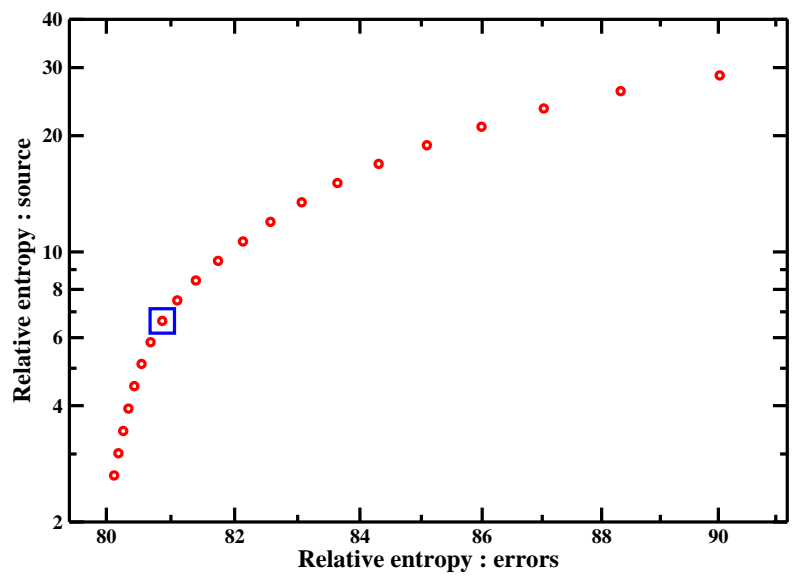

Fig. 4. L-curve for a varying mass with an inflection point identified as $m=8$ (square). Results obtained for a spatial resolution of $2.25^{\circ} \times 2.25^{\circ}$.

$$
\begin{aligned}
\bar{\sigma}_{k} & =m_{k} \frac{\gamma_{k} \exp \left(m_{k}\left[\overline{\boldsymbol{\beta}}^{\dagger} \boldsymbol{H}\right]_{k}\right)}{1-\gamma_{k}+\gamma_{k} \exp \left(m_{k}\left[\overline{\boldsymbol{\beta}}^{\dagger} \boldsymbol{H}\right]_{k}\right)}, \\
\overline{\boldsymbol{\varepsilon}} & =\boldsymbol{R} \overline{\boldsymbol{\beta}}
\end{aligned}
$$

Note that $m_{k}$ is a mass scale that is taken constant on all grid-cells, $m_{k} \equiv m . \gamma_{k}$ is the probability that a release occurs in cell $k$, which is also taken constant, $\gamma_{k} \equiv \gamma$, and set to a very low value. This prior assumption for the source allows for a single grid-cell source (most probable case) but also for a multiple grid-cell source.

\subsubsection{L-curve estimates}

The prior probability density functions, $\nu(\boldsymbol{\sigma})$ and $\zeta(\boldsymbol{\varepsilon})$, are defined with the help of some parameters whose values would often be set more or less arbitrarily according to experience, rather than to some objective criteria. In particular, for Bernoulli law, the emitted mass $m$ is an example of such a parameter. Similarly, Gaussian distribution describing errors is parametrised with an arbitrary value of the standard deviation $\sqrt{\chi}$. Therefore, in the first place, we establish the optimal values of those parameters. Due to nonlinearity of the dependence of the cost function on $m$, the analysis has been undertaken with the L-curve technique. The technique was introduced in (Hansen, 1992), and its application to source inversion is detailed in (Davoine and Bocquet, 2007).

Firstly, a standard deviation uniform for all the measurements, and hence giving more weight to large values, has been chosen equal to $\sqrt{\chi}=0.3 \mathrm{ng} \mathrm{m}^{-3}$, according to (Bocquet, 2007). While varying $m$ within a large interval of its values, we have checked for the one corresponding to the Lcurve corner. It turned out to be $m=10 \times M / \lambda^{2}$, where $M$ 
is the true quantity of the released PMCP during a one-hour interval and $\lambda$ is a parameter that sets the space scale (arbitrarily equal to 4 and 8 for the resolution $2.25^{\circ} \times 2.25^{\circ}$ and $4.5^{\circ} \times 4.5^{\circ}$, respectively).

Then, having set $m=10$ (dropping the conventional unit $M / \lambda^{2}$ ), we have applied the procedure again, this time for a varying standard deviation. The point of inflection has been obtained for $\sqrt{\chi}=0.25 \mathrm{ng} \mathrm{m}^{-3}$, Fig. 3. Next, an L-curve experiment with a varying $m$ has been repeated, this time for the fixed $\sqrt{\chi}=0.25 \mathrm{ng} \mathrm{m}^{-3}$. It let us identify $m=8$ as the point of inflection, Fig. 4. At this point the iterative procedure has already converged to the optimal values of $m$ and $\chi$.

\section{Inversion results for ETEX-II}

We present in this section the inversion results obtained for the ETEX-II experiment.

\subsection{Plain results}

Figure 6 shows the inverted profile in Monterfil (in the grid-cell containing Monterfil) for the spatial resolution of $2.25^{\circ} \times 2.25^{\circ}$ and temporal resolution of $1 \mathrm{~h}$, while Fig. 5 illustrates spatial distribution of the inverted source which has been integrated in time. The reconstructed mass in Monterfil is equal to $51 \mathrm{~kg}$ and the total reconstructed mass is $73 \mathrm{~kg}$.

If we coarsen the spatial resolution of the domain of inversion, $4.5^{\circ} \times 4.5^{\circ}$, then the inverted mass in Monterfil is equal to $66 \mathrm{~kg}$ and the total reconstructed mass sums up to $87 \mathrm{~kg}$. On the other hand, inversion on the $1.125^{\circ} \times 1.125^{\circ}$ grid fails but, as shown in (Bocquet, 2005b), such a failure is bound to occur for a fine grid.

3.1.1 Marginal gain of information provided by each observation

The assimilation of the data through the source inversion process results in a net gain of information accumulated by the optimality system. What the observations convey to the optimality system is measured by a relative entropy number denoted $\mathcal{K}_{\boldsymbol{\sigma}}$, Eq. (3). In a general, non-Gaussian case, a single measurement contribution to the gain $\mathcal{K}_{\sigma}$ depends on the solution and hence on the actual values of the other measurements. That is why one investigates the marginal contribution $\partial_{\mu_{i}} \mathcal{K}_{\sigma}$ of an observation $\mu_{i}$, for all the observations. In (Bocquet, 2008), it was shown how to compute such a marginal contribution. These contributions have also been computed for ETEX-II and are plotted in Fig. 7.

Moreover, the Lagrange multipliers $\boldsymbol{\beta}$ have been pictured. They represent the marginal total gain of information (which can either serve to identify the source or be lost in the deblurring of the errors in the data): $\partial_{\mu_{i}} \mathcal{K}_{\sigma, \boldsymbol{\varepsilon}}$. They are also a direct measure of how sensitive the optimality system is to each measurement seen as a constraint. Finally, because

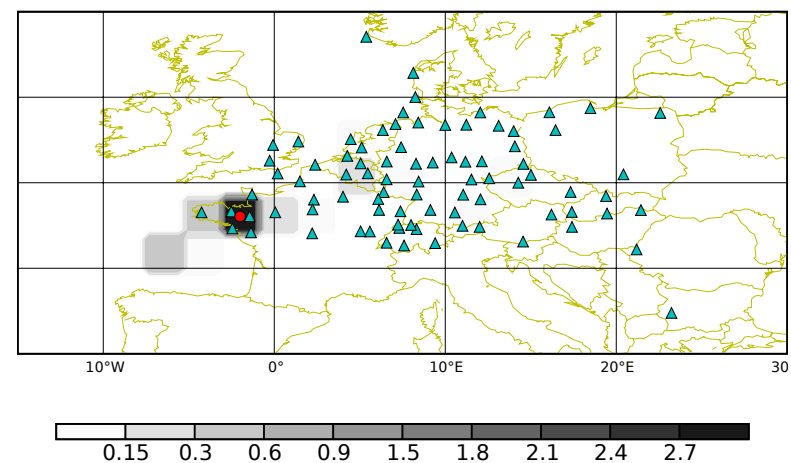

Fig. 5. ETEX-II. Total reconstructed mass in each spatial grid-cell for the resolution of $2.25^{\circ} \times 2.25^{\circ}$. The triangles represent the monitoring stations which gathered the measurements used in the inversion. The disc marks the true release site, Monterfil, France. The units are in $\mathrm{kg}$.

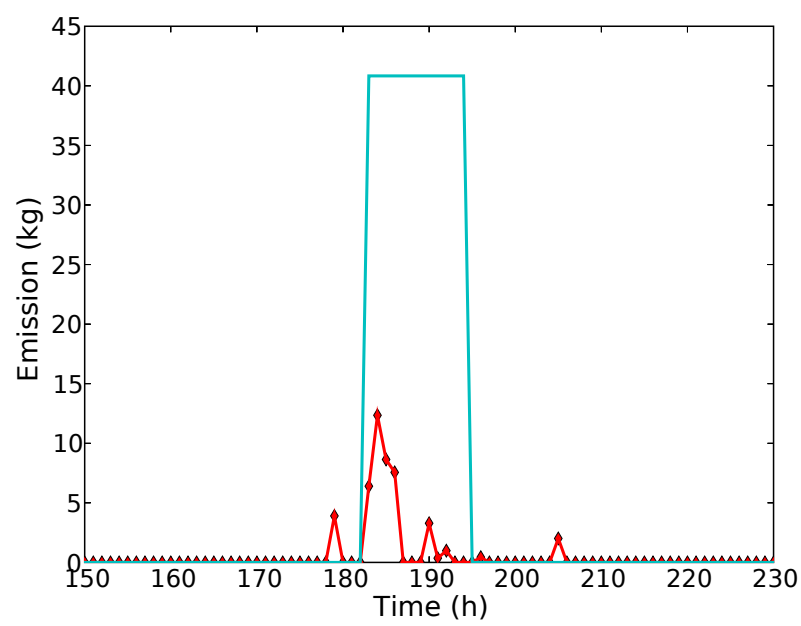

Fig. 6. ETEX-II. Profile of the reconstructed source in Monterfil. Total reconstructed mass is equal to $73 \mathrm{~kg}$ and the mass reconstructed in Monterfil is $51 \mathrm{~kg}$. Spatial resolution is $2.25^{\circ} \times 2.25^{\circ}$.

the prior hypothesis on the errors is Gaussian, they are also directly proportional to the diagnosed errors $(\overline{\boldsymbol{\varepsilon}}=\boldsymbol{R} \overline{\boldsymbol{\beta}})$.

From Fig. 7, one learns that the diagnosed errors depend strongly on most measurements in France and Benelux: a change in a measurement results in an increase of the errors, rather than a new piece of information on the source. This contrasts with the analogue result for ETEX-I (Bocquet, 2008). Nor is it the case for ETEX-II data coming from Central and Eastern Europe. The information that is gained on the source when perturbing the latter measurements is rather strong (again as compared to ETEX-I). This can be explained by the fact that the plume moved eastwards rapidly so that these observations are more informative than for ETEX-I. But also by the fact that the information that they bring about 

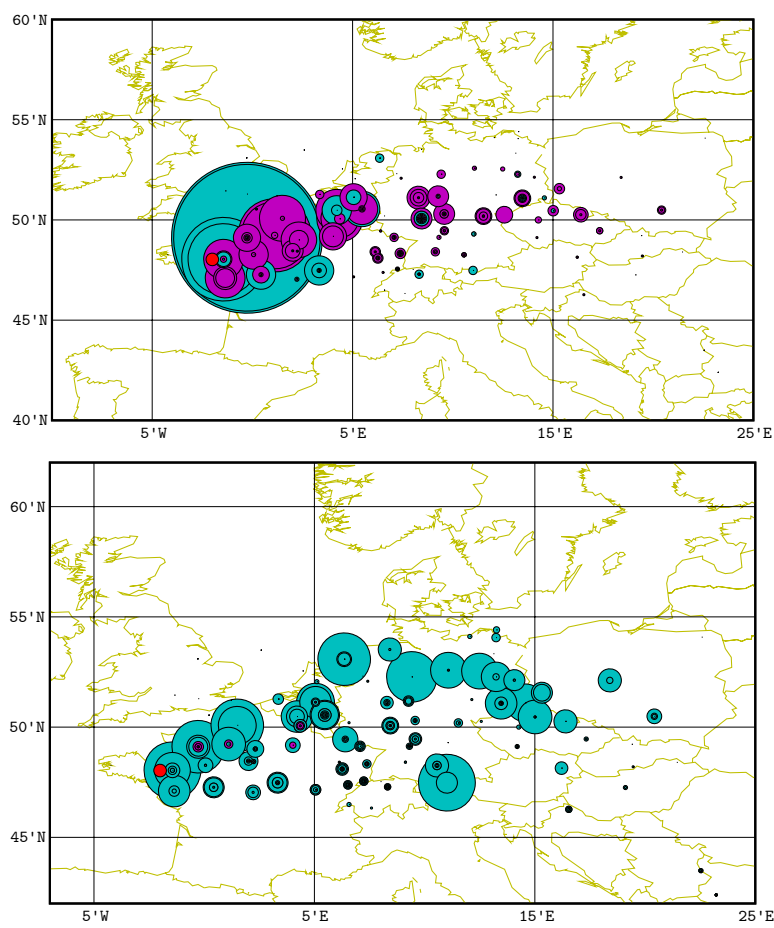

Fig. 7. ETEX-II. Marginal contributions of each observation to the total information gained in the assimilation $\mathcal{K}_{\boldsymbol{\sigma}, \boldsymbol{\varepsilon}}$ (top figure), and for the information gained on the source alone $\mathcal{K}_{\sigma}$ (bottom figure). The disc radius is proportional to the value of the sensitivity with respect to the measurement. Darker discs correspond to negative values.

on the source is more trusted than the information got from the Western measurements (at least relatively to the similar ETEX-I analysis). So that, compared with ETEX-I, the optimality system trusts more the Eastern observations, which were probably found more consistent than the Western ones.

\subsection{Comparison to ETEX-I}

We report here inversion results that have been obtained for the ETEX-I experiment (Bocquet, 2007), but improved by parameters' estimation. In the first ETEX release $340 \mathrm{~kg}$ of $\mathrm{PMCH}$ were emitted to the atmosphere. Before source inversion has been addressed, a similar L-curve analysis has been performed. Consequently, an entire interval of the values of $m$ and $\chi$ ensuring robust inversions has been identified. The borders of the domain of those values are given by $\left[m=5 ; \chi=0.30 \mathrm{ng} \mathrm{m}^{-3}\right] \times\left[m=12 ; \chi=0.45 \mathrm{ng} \mathrm{m}^{-3}\right]$, where $m$ is in units of the truly released quantity of PMCH per hour. The reconstructed mass in Monterfil is $244 \mathrm{~kg}$ and the total reconstructed mass is equal to $344 \mathrm{~kg}$ for the lower boundaries and, respectively, $224 \mathrm{~kg}$ and $336 \mathrm{~kg}$ for the upper boundaries of the domain.

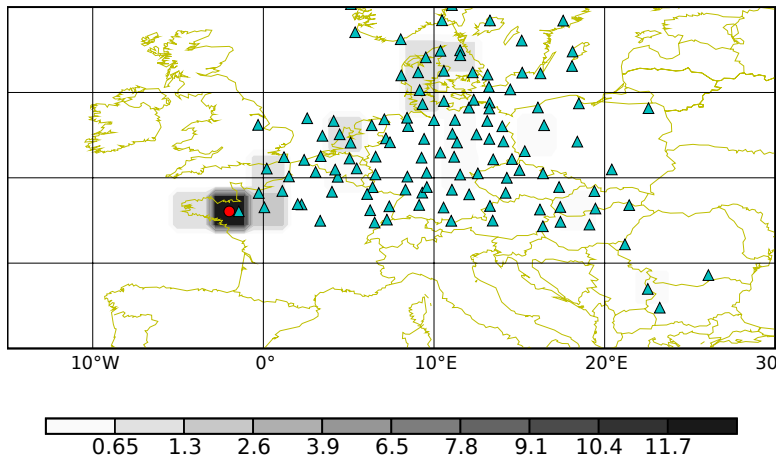

Fig. 8. ETEX-I. Total reconstructed mass in each spatial grid-cell for the resolution of $2.25^{\circ} \times 2.25^{\circ}$. The triangles represent the monitoring stations which gathered the measurements used in the inversion. The disc marks the true release site. The units are in $\mathrm{kg}$.

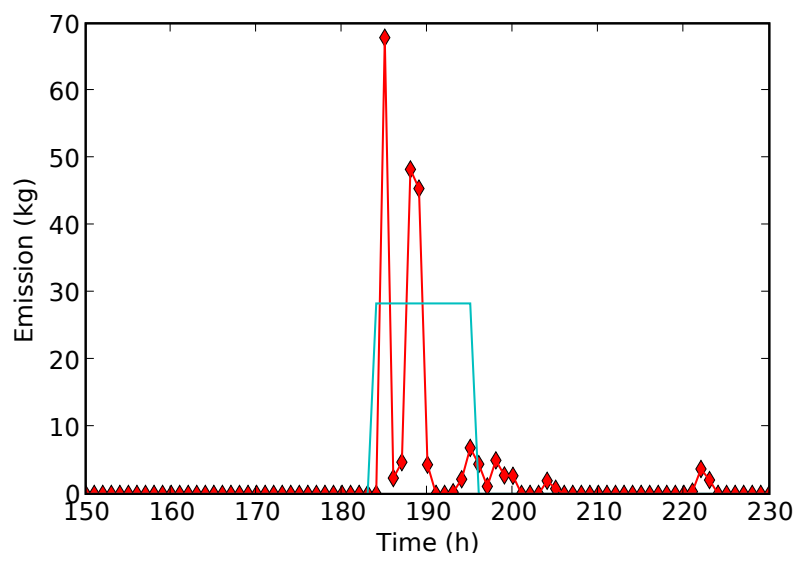

Fig. 9. ETEX-I. Profile of the reconstructed source in Monterfil. Total reconstructed mass is equal to $336 \mathrm{~kg}$ and the mass reconstructed in Monterfil is $224 \mathrm{~kg}$. Spatial resolution is $2.25^{\circ} \times 2.25^{\circ}$.

A noteworthy feature of the ETEX-I inversion is also the fact that, contrary to the ETEX-II results, the method lets invert the source on both coarse, $2.25^{\circ} \times 2.25^{\circ}$, and fine grid, $1.125^{\circ} \times 1.125^{\circ}$.

In order to enable comparison with the ETEX-II retrievals we show in Fig. 9 a reconstructed profile corresponding to the one given in Fig. 6 and the spatial distribution of the reconstructed mass in Fig. 8, an analogue of Fig. 5.

\section{Discussion of the results}

The localisation of the ETEX-II source is rather good. $71 \%$ of the recovered mass is attributed to the correct grid-cell, as compared to $65 \%$ in the ETEX-I case. This can be explained 
by the optimality system to deblur some of the data. It is also explained by a very high sensitivity of the source to the nearby measurements which makes the Monterfil grid-cell a very likely spot. Nevertheless, the reconstructed mass accounts only for a small fraction of the truly released tracer, and implies the necessity of tackling the question of possible reasons behind this result.

\subsection{Meteorological conditions}

The meteorological situation was similar for both ETEX experiments with respect to the main feature structuring the atmospheric circulation - a low pressure system located over the British Isles.

At the release site, according to the figures in (Gryning et al., 1998), the surface layer was well mixed-up during the first part of the release, and stably stratified later on. This statement is valid for both releases, the height of the mixedlayer being smaller in the ETEX-II case. In contrast to the first release, the wind direction in the second one was roughly constant, and horizontal wind speed, although decreasing, remained greater than $5 \mathrm{~m} \mathrm{~s}^{-1}$. Hence, up to the time of the passage of a cold front during the second release, the meteorological situation at the release site seemed to be fostering a correct model-measurement comparison, even more for the second experiment than for the first one.

Away from the release site, the conditions were also favourable. According to (Stohl and Koffi, 1998), where model trajectories based on the ECMWF analysed wind fields have been compared to the balloon tracks: "the ECMWF fields of the horizontal wind were of exceptionally good quality in the second experiment". Further on: "Since the horizontal winds are balanced by the vertical winds, this also gives an indication that the grid scale vertical winds were not too bad." Thus, most probably, the advection was correctly accounted for in the transport models.

Less than an hour before the end of the second release, a cold front crossed the release site. During the two hours preceding the passage of the front, according to (Gryning et al., 1998): "an uninterrupted vertical wind in the layer between the ground and up to $300 \mathrm{~m}$ (the maximum range of the SODAR during this period) is measured". The front must have crossed the tracer plume before crossing the release site. According to (Stohl and Koffi, 1998), due to plume's travelling long enough "almost along this front" there was a possibility of an uplift into the free troposphere "by organized rising motion ahead of the front or by convective processes behind the front".

However, some arguments against convective rise of the tracer can also be found. At the release site, the front seems not to be accompanied by a change in a heat flux, see Fig. 12 in (Gryning et al., 1998). At least not before the following day which reflects typical diurnal/nocturnal variability of the heat flux and is less pronounced than for the first release, Fig. 6 in (Gryning et al., 1998). Moreover, according to
(Ryall and Maryon, 1998): "The observations of mainly light rain and drizzle in the general area do not support the presence of vigorous convective cloud, but the dynamics were indeed vigorous, and it seems likely that, at least in part, frontal uplift may have accounted for the dilution."

At this stage, the most important source of model error, seems to be a presumable uplift of the tracer due to "unresolved small-scale vertical winds in the vicinity of the cold front" (Stohl and Koffi, 1998). However, its 2-h duration excludes, by far, its supposed blame for eliminating $85 \%$ of the tracer at the release site. Even more as, although at different stages of the release interval, the vertical movements of similar intensity were also observed at the release site during the first release. Hence, should the meteorological conditions be to be blamed for the missing tracer, it would have been mostly eliminated by the localised vertical motions crossing the plume away from the release site.

\subsection{Inverse modelling contribution}

How does inverse modelling tackle errors, in particular an error linked to the presumable phenomenon of small-scale vertical winds? First of all, it should be emphasised here that inverse modelling tends to do more than running a model backwards. In particular, like for any proper data assimilation scheme, our inverse modelling approach inverts both the source and the errors. The source estimation, via the observation equation given by Eq. (1), is thus not a straightforward inference from the measurements through the model. It rather obeys to the normal equations that balance information on the source and information on the errors, according to their respective uncertainty:

$$
\begin{aligned}
& \overline{\boldsymbol{\sigma}}=\boldsymbol{B} \boldsymbol{H}^{\dagger}\left(\boldsymbol{R}+\boldsymbol{H} \boldsymbol{B} \boldsymbol{H}^{\dagger}\right)^{-1} \boldsymbol{\mu}, \\
& \bar{\varepsilon}=\boldsymbol{R}\left(\boldsymbol{R}+\boldsymbol{H} \boldsymbol{B} \boldsymbol{H}^{\dagger}\right)^{-1} \boldsymbol{\mu} .
\end{aligned}
$$

For clarity reasons, these particular formulae use Gaussian assumptions on both the source and the error. The former one is parametrised here with the background error covariance matrix $\boldsymbol{B}$, an analogue of the observation error covariance matrix $\boldsymbol{R}$ parametrising Gaussian errors $\boldsymbol{\varepsilon}$. The formulae used in the inversion procedure, Eq. (5), also rely on Gaussian modelling of errors but the prior source over there is described with the Bernoulli law.

Such a Gaussian prior should account for a local, uncorrelated, additive, moderately large error. In particular, uncorrelated instrumental errors and subgrid model errors, like the above mentioned small-scale vertical motions crossing the plume away from the release site, should, at least partially, be accounted for in the procedure. However, as illustrated in Fig. 10, the measurements arising from the reconstructed source are largely scattered as a function of the real measurements. No improvement in consistency due to error modelling could have been observed. Hence, the inverse 


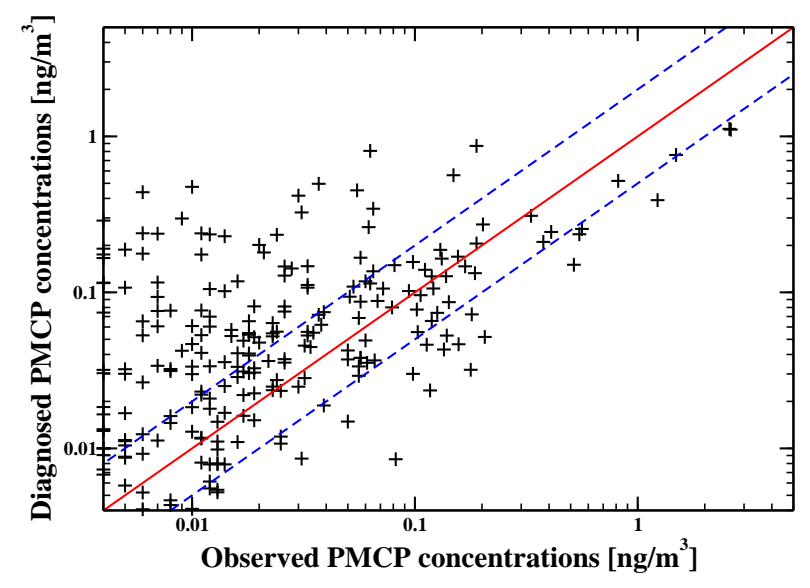

Fig. 10. Posterior measurement consistency test. Measurements (diagnosed PMCP) simulated from the reconstructed source (Fig. 6) as a function of the real measurements (observed PMCP). The dashed lines mark the discrepancy factor equal to 2 .

modelling analysis of the ETEX-II data does not confirm the hypothesis that has been made on the errors.

Indeed, the inconsistency of the measurements could have already been noticed in a data-simulation comparison. In the present studies, however, the reconstruction method takes into account a physical model, and both the source and the errors have been retrieved. Thus, the detected inconsistencies go beyond statistical ones which could be diagnosed through geostatistical procedures (Dubois et al., 2005).

There are two possible explanations for these inconsistencies. Firstly, there are obviously limitations to independent, Gaussian-modelled errors. Large-scale motions that are not accounted for by the model are to evade the inverse modelling analysis. It is thus clear that if most of the mass had been lifted upward, above the inversion cap, right from the beginning at the release site, it could not have been retrieved with an inverse modelling system based on a CTM without convection schemes, like ours. Such a case, however, seems to be excluded by the analysis of the meteorological conditions. Moreover, the remaining mass trapped in the boundary layer could have displayed a stronger consistency once inverse modelling has been performed but we have not exhibited such a phenomenon. Thus, secondly, the inconsistencies may suggest that there is a systematic error in the collected measurements.

\section{Conclusions}

We have recalled here the results of source retrieval in the case of the ETEX-I release. The reconstruction performed with the method of maximum entropy has been shown to render satisfactory results in this case. The same procedure employed in source retrieval of the ETEX-II release gives much worse results. The official report on ETEX-II states that 75\% of the mass is unaccounted for from the measurements. Our study rises this level up to $85 \%$, as if the observed concentrations had been diluted by an overall mean factor of 6.8. One could point several reasons for the difficulties encountered by the inversion procedure in this case.

Firstly, the number of the measurements is limited what has an impact on the attainable source resolution (coarser than for ETEX-I). Secondly, the quality of the inversion is highly dependent on the possibility of incorporating error into the modelling procedure. Because convection is not properly taken into account, merely fronts, a sudden uplift of the plume might have been missed resulting in an important model error and a loss of mass not reproduced in the modelling. However, a closer look at the meteorological conditions implies that the most important sources of error were due to some presumable small-scale vertical motions not resolved in the meteorological fields. These motions were supposed to accompany the cold front on its crossing of the plume. If the errors of this form were indeed reflected in the measurements they should, partly at least, be corrected by the inverse modelling procedure. The lack of such an improvement contradicts the initial hypothesis on the errors' features. Moreover, a general experience from chemistry transport model validation against all possible measurements (as e.g. the EMEP data in Europe) provides an additional argument against the vertical movements being responsible for such an important mass' loss. Validation procedure requires an important number of simulations where frequent front passages occur. This implies that the frontal uplift situation should influence the model results frequently and that very often the model results should be biased by a factor of up to ten. This is, however, not the case. Some attempts to explain the inconsistencies in the measurements, like the presence of an additional source emitting after the end of the release, have also been made (Bellasio et al., 2000). Nevertheless, no indication of the presence of such a supplementary source has been detected in the inverse modelling studies.

These arguments cast doubt on the quality and/or representativeness of the measurements. From the reconstruction point of view, the measurements seem inconsistent, in particular the observations performed in the early evolution of the plume (over France and Benelux). But other clues, independent from this study, also plead against the data. Although PMCP concentrations extracted from the collected samples are of good quality, the samples themselves are more difficult to judge. In many stations cloud's presence was intermittent. In particular, several cases are reported in which some stations exhibit cloud passage twice during the sampling period while another station, located close by, misses the second peak. Hence, the measurements suggest plume was chopped into several clouds which moved separately with different velocities (Nodop, 1998). This behaviour is, however, not supported by any of the models involved in model intercomparison (Graziani et al., 1998b). Moreover, the aircraft data 
(Girardi et al., 1998) do not reveal any splitting of the plume. In addition, even though it has been argued against any water dilution of the samples, it has also been mentioned (Girardi et al., 1998) that the instruments' catalyst (Palladium) have been repeatedly poisoned during the analytical campaign by an unknown compound, so that the catalyst needed replacement several times.

Acknowledgements. The authors would like to thank three anonymous referees for their remarks which resulted in improving the arguments behind paper's conclusion. The authors are grateful to Roberto Bellasio for his very kind and quick response, and for the copy of his paper.

Edited by: S. Galmarini

\section{References}

Bellasio R., Bianconi R., Mosca S., Girardi F., Graziani G., and Klug W.: Simulation of the second ETEX release in the proximity of the source. Int. J. Environment and Pollution, 14, 296-306, 2000.

Bocquet, M.: Reconstruction of an atmospheric tracer source using the principle of maximum entropy. I: Theory, Q. J. R. Meteorol. Soc, 131, 2191-2208, 2005.

Bocquet, M.: Grid resolution dependence in the reconstruction of an atmospheric tracer source, Nonlin. Processes Geophys., 12, 219-234, 2005, http://www.nonlin-processes-geophys.net/12/219/2005/.

Bocquet, M.: Reconstruction of an atmospheric tracer source using the principle of maximum entropy. II: Applications, Q. J. R. Meteorol. Soc, 131, 2209-2224, 2005.

Bocquet, M.: High resolution reconstruction of a tracer dispersion event: application to ETEX, Q. J. R. Meteorol. Soc, 133, 10131026, 2007.

Bocquet, M.: Inverse modelling of atmospheric tracers: NonGaussian methods and second-order sensitivity analysis, Nonlin. Processes in Geophys., 15, 127-143, 2008.

Brandt, J.: Modelling Transport, Dispersion and Deposition of Passive Tracers from Accidental Releases, PhD thesis, National Environmental Research Institute, Roskilde, Denmark, 307 pp., 1998.

Brandt, J., Bastrup-Birk, A., Christensen, J. H., Mikkelsen, T., Thykier-Nielsen, S., and Zlatev, Z.: Testing the importance of accurate meteorological input fields and parameterizations in atmospheric transport modelling, using DREAM - validation against ETEX-1, Atmos. Env., 32, 4167-4186, 1998.
Davoine, X. and Bocquet, M.: Inverse modelling-based reconstruction of the Chernobyl source term available for long-range transport, Atmos. Chem. Phys, 7, 1549-1564, 2007.

Dubois, G., Galmarini, S., and Saisana, M.: Geostatistical investigation of ETEX-1: Structural analysis, Atmos. Env., 39, 16831693, 2005.

Girardi, F., Graziani, G., van Velzen, D., Galmarini, S., Mosca, S., Bianconi, R., Bellasio, R., Klug, W., and Frase, G.: The Euopean Tracer Experiment, EUR 18143 EN, Office for Official Publications of the European Communities, Luxembourg, 107 pp., 1998.

Graziani, G., Galmarini, S., Grippa, G., and Klug, W.: Real-time long-range dispersion model evaluation of ETEX second release, EUR 17755 EN, Office for Official Publications of the European Communities, Luxembourg, 302 pp., 1998.

Graziani, G., Klug, W., and Mosca, S.: Real-time long-range dispersion model evaluation of ETEX first release, EUR 17754 EN, Office for Official Publications of the European Communities, Luxembourg, 213 pp., 1998.

Gryning, S.-E., Batchvarova, E., Schneiter, D., Bessemoulin, P., and Berger, H.: Meteorological conditions at the release site during the two tracer experiments, Atmos. Env., 32, 4123-4137, 1998.

Hansen, C.: Analysis of discrete ill-posed problems by means of the L-curve, SIAM Review, 34, 561-580, 1992.

Krysta, M. and Bocquet, M.: Source reconstruction of an accidental radionuclide release at European scale, Q. J. R. Meteorol. Soc., 133, 529-544, 2007.

Mosca, S., Bianconi, R., Bellasio, R., Graziani, G., and Klug, W.: ATMES II - evaluation of long-range dispersion models using data of the 1st ETEX release, EUR 17756 EN, Office for Official Publications of the European Communities, Luxembourg, 459 pp., 1998.

Nodop, K. : ETEX Symposium on long-range atmospheric transport model verification and emergency response, 1316 May 1997, Vienna (Austria), Proceedings, 252 pp., 1997.

Nodop, K., Connolly, R., and Girardi, F.: The field campaigns of the European tracer experiment (ETEX): overview and results, Atmos. Env., 32, 4095-4108, 1998.

Quélo, D., Krysta, M., Bocquet, M., Isnard, O., Minier, Y., and Sportisse, B.: Validation of the Polyphemus platform on the ETEX, Chernobyl and Algeciras cases, Atmos. Env., 41, 53005315, 2007.

Ryall D. B. and Maryon, R. H.: Validation of the UK Met. Office's NAME Model against the ETEX dataset, Atmos. Env., 32, 42654276, 1998.

Stohl A. and Koffi, N. E.: Evaluation of trajectories calculated from ECMWF data against constant volume balloon flights during ETEX, Atmos. Env., 32, 4151-4156, 1998. 